\title{
A Subwavelength Plasmonic Waveguide Filter with a Ring Resonator
}

\author{
Xiang Zhai, ${ }^{1,2}$ Shuangchun Wen,, ${ }^{1,2}$ Dong Xiang, ${ }^{2,3}$ Ling-Ling Wang, \\ Wujiaihemaiti Rexidaiguli, ${ }^{2}$ Liu Wang, ${ }^{2}$ and Dianyun Fan $^{1,2}$ \\ ${ }^{1}$ Key Laboratory for Micro/Nano Optoelectronic Devices of Ministry of Education, School of Information Science and Engineering, \\ Hunan University, Changsha 410082, China \\ ${ }^{2}$ School of Physics and Microelectronics, Hunan University, Changsha 410082, China \\ ${ }^{3}$ School of Nuclear Science and Technology, University of South China, Hengyang 421001, China
}

Correspondence should be addressed to Shuangchun Wen; scwen@hnu.edu.cn and Dong Xiang; xiangdong007@163.com

Received 29 January 2013; Accepted 20 February 2013

Academic Editor: Bo Yu

Copyright (C) 2013 Xiang Zhai et al. This is an open access article distributed under the Creative Commons Attribution License, which permits unrestricted use, distribution, and reproduction in any medium, provided the original work is properly cited.

\begin{abstract}
The transmission characteristics of the electromagnetic wave are numerically investigated in two-dimensional compound plasmonic structures composed of two straight subwavelength metal-insulator-metal (MIM) waveguides and a ring resonator. The two straight MIM waveguides situate on both sides of the ring resonator, and the MIM waveguide of the outgoing side has a positional angular deviation $\theta$ relative to the MIM waveguide of the incoming side. The results show that the filtering performances of the asymmetric structures $\left(\theta \neq 0^{\circ}\right)$ are greatly improved in comparison with the symmetric structure $\left(\theta=0^{\circ}\right)$. For most of the asymmetric structures, there is a transmission minimum at the slightly bigger wavelength than one at which the transmission peak appears for the symmetric structures, and there is still a transmission peak at the wavelength of the transmission peak of the symmetric structures. Moreover, the difference between the transmission peak and valley is increased, and the breadth of the transmission peak becomes narrow.
\end{abstract}

\section{Introduction}

Surface plasmon polaritons (SPPs) are p-polarized optical surface waves that propagate along a metal-dielectric interface with fields that peak at the interface and decay exponentially away into both sides [1]. However, the single metal-dielectric interface SPP, characterized by high attenuation and low confinement, limits the scope for applications. To date, one of the most practical ways of enhancing the SPP confinement is to use a metal-insulator-metal (MIM) subwavelength waveguide structure, along which a symmetrically coupledSPP mode can propagate. This provides us an avenue to concentrate and channel light with subwavelength structures and develop practical nanophotonic devices for future information technologies. Thus, some novel plasmonic elements based on MIM waveguide structures have been proposed, such as bends and splitters [2], Y-shaped combiners [3], Mach-Zehnder interferometers $[4,5]$, Bragg reflectors [6,
7], directional couplers [8,9], and tooth-shaped plasmonic waveguide filters [10].

Recently, Wang and his coworkers [11] have investigated two-dimensional (2D) compound plasmonic structures composed of two straight MIM waveguides with a ring resonator. Their result shows that some transmission resonance peaks appear at some specific wavelengths which meet the resonance conditions in the ring. However, the discussed compound structure in their work is symmetric; that is, both of the two MIM straight waveguides are located symmetrically at both sides of the ring resonator. This suggests that even if the resonance condition has not been satisfied, optical wave will still have not low transmission. Therefore, if the symmetrical distribution is broken, the properties of the optical transmission through this kind of asymmetric structure can present more novel features, and then the filtering performance will be greatly improved. In this work, we will mainly discuss the transmission characteristics of 
the asymmetric structure based on MIM plasmonic waveguides with a ring resonator.

\section{Model and Method}

Figure 1 shows schematically a 2D subwavelength plasmonic waveguide filter composed of two straight MIM waveguides and a ring resonator. The two MIM waveguides situate on both sides of the ring resonator, and the MIM waveguide of the outgoing side (waveguide II) has a positional angular deviation $\theta$ relative to the MIM waveguide of the incoming side (waveguide I). All the widths of the slits in the two MIM waveguides and the width of the ring are $w=50 \mathrm{~nm}$. The radius of the ring is $r$, which is the average of the inner radius $r_{i}$ and the outer radius $r_{a}, r=\left(r_{a}+r_{i}\right) / 2$. The medium of the the slits and the ring (white area) is assumed to be air whose relative permittivity $\varepsilon_{d}$ is set to be 1 . The metal (gray area) is chosen to be silver, whose frequency-dependent relative permittivity obeys the Drude model as $\varepsilon_{m}(\omega)=\varepsilon_{\infty}-\omega_{p}^{2} /\left(\omega^{2}+\right.$ $i \gamma \omega)$. Here $\varepsilon_{\infty}=4.2$ is the high-frequency bulk permittivity, $\omega_{p}=1.346 \times 10^{16} \mathrm{rad} / \mathrm{s}$ is the bulk plasmon frequency, $\gamma=$ $9.617 \times 10^{13} \mathrm{rad} / \mathrm{s}$ is the damping factor, and $\omega$ is the angular frequency of the incident electromagnetic radiation [12].

The 2D finite-difference time-domain (FDTD) method [13] is employed to simulate and calculate the optical transmission through these gratings. In our simulation, the spatial mesh steps are set $\Delta x=\Delta z=5 \mathrm{~nm}$ and the time step is set $\Delta t=\Delta x / 2 c$ ( $c$ is the velocity of light in the air), and the calculated region is truncated by using Mur boundary condition on all the boundaries. Only normally incident $p$-polarized waves are considered here, implying that the magnetic field $(H)$ is along the $y$ direction. Two power monitors are set at the locations Port-1 and Port-2, far from the ring $150 \mathrm{~nm}$, to detect the incident and the transmission fields for calculating the incident power of $P_{\text {in }}$ and the transmitted power of $P_{\text {out }}$, respectively. The transmittance is defined to be $T=P_{\text {out }} / P_{\text {in }}$.

\section{Simulation Results and Discussion}

Firstly, we initialized the radius of the ring $r=155 \mathrm{~nm}$ and studied the effect of the positional angular deviation $\theta$ on transmission spectrum of the structure. Figure 2 displays the calculated transmission spectra of the structure with different $\theta\left(0^{\circ}, 30^{\circ}, 45^{\circ}, 60^{\circ}\right.$, and $\left.90^{\circ}\right)$ at the range from $400 \mathrm{~nm}$ to $1800 \mathrm{~nm}$, respectively. In Figure 2, one can find the following main features. First, the transmission spectra of the asymmetric structures $\left(\theta \neq 0^{\circ}\right)$ are almost the symmetric structure $\left(\theta=0^{\circ}\right)$, and the transmittances of symmetrical structure are approximately maxima at all the wavelength range. Second, when $\theta=0^{\circ}$, there are three transmission maxima corresponding to $457 \mathrm{~nm}, 665 \mathrm{~nm}, 1235 \mathrm{~nm}$ and two minima located at $523 \mathrm{~nm}$ and $861 \mathrm{~nm}$. However, these transmission minima are still relatively high and greater than 0.5 , even the difference between the transmission peak and valley is only about 0.4 . Third, for the most of the asymmetric structures $\left(\theta \neq 0^{\circ}\right)$, a transmission minimum appears at the slightly bigger wavelength than one at which the transmission peak

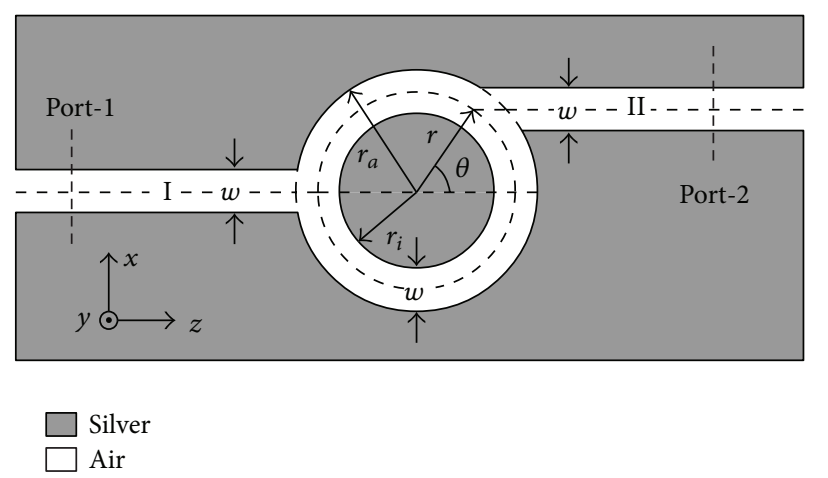

FIGURE 1: Schematic of the MIM plasmonic waveguide with a ring resonator.

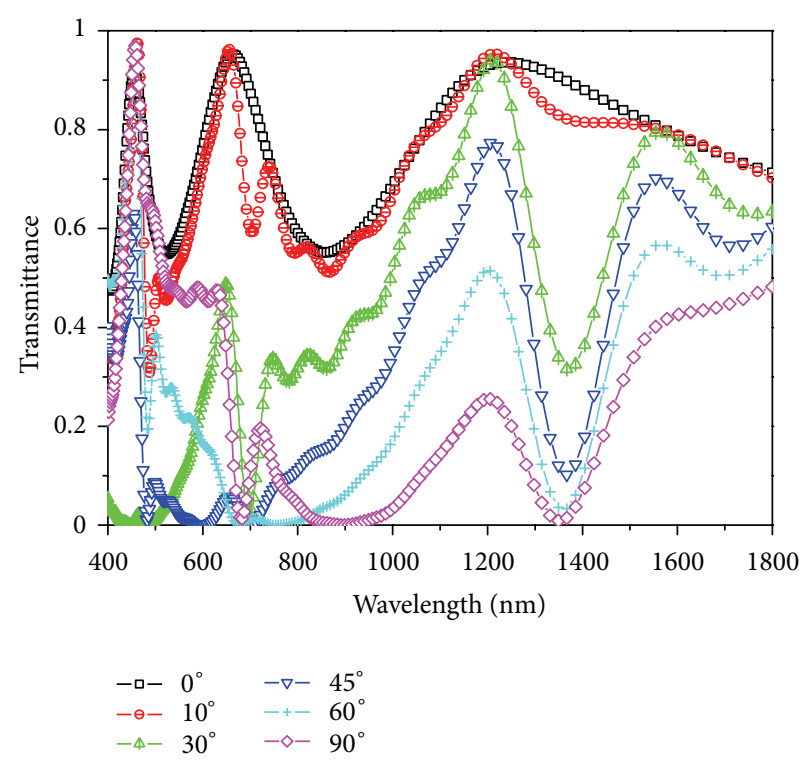

Figure 2: (Color Online) Transmission spectra of the MIM plasmonic waveguide with a ring resonator and different $\theta$ for $r=$ $155 \mathrm{~nm}$.

appears for the symmetric structure $\left(\theta=0^{\circ}\right)$, and some of these transmission minima are almost reduced to zero. For example, the transmission minima are almost zero at wavelengths $488 \mathrm{~nm}$ and $697 \mathrm{~nm}$ for $\theta=30^{\circ}, 483 \mathrm{~nm}$ and $702 \mathrm{~nm}$ for $\theta=45^{\circ}, 478 \mathrm{~nm}$ and $1367 \mathrm{~nm}$ for $\theta=60^{\circ}$, and $683 \mathrm{~nm}$ and $1332 \mathrm{~nm}$ for $\theta=90^{\circ}$. Fourth, for the most of the asymmetric structures $\left(\theta \neq 0^{\circ}\right)$, there is still a transmission peak at the peak wavelength of the symmetric structure $\left(\theta=0^{\circ}\right)$, but the transmittance becomes smaller. Here, it should be pointed out that the transmittance at wavelength about $1200 \mathrm{~nm}$ decreases gradually as the positional angular deviation $\theta$ increases from $0^{\circ}$ to $90^{\circ}$. Finally, just because of the above two, when $\theta \neq 0^{\circ}$, the breadth of the transmission peak becomes narrow and the difference between the transmission peak and valley is increased apparently. For example, when $\theta=45^{\circ}$, transmission maximum at wavelength $1206 \mathrm{~nm}$ is about 0.77 , while the transmission minima at wavelengths $702 \mathrm{~nm}$ and $1367 \mathrm{~nm}$ are about 0.002 and 0.1 , respectively, so the difference 
between the transmission peak and valley increases to about 0.7 .

It is very clear that the transmission characteristics of these above MIM plasmonic waveguide structures with a ring resonator mainly depend on two kinds of factors: one is electromagnetic wave resonance effect of the ring cavity, and the other is the relative positions of two MIM waveguides I and II on both sides of the ring. It is known that the resonating wavelength of a ring resonator can be obtained theoretically by the following equation:

$$
n_{\mathrm{eff}} \cdot 2 \pi r=N \cdot \frac{\lambda}{2}
$$

where $n_{\text {eff }}$ is the effective index of the coupled-SPP mode in the ring, $\lambda$ is vacuum wavelength of the incident wave, and $N$ is mode number. Because the light channel in the ring can be approximated as arc-shaped MIM waveguide, for $p$-polarized case, $n_{\text {eff }}$ can be approximately calculated by the following [14]:

$$
\frac{\varepsilon_{d} \sqrt{n_{\mathrm{eff}}^{2}-\varepsilon_{m}}}{\varepsilon_{m} \sqrt{n_{\mathrm{eff}}^{2}-\varepsilon_{d}}}=\frac{1-\exp \left(w \sqrt{n_{\mathrm{eff}}^{2}-\varepsilon_{d}}\right)}{1+\exp \left(w \sqrt{n_{\mathrm{eff}}^{2}-\varepsilon_{d}}\right)} .
$$

Secondly, in order to show electromagnetic wave resonance effect of ring cavity, we calculated the electromagnetic field distribution of the three transmission maxima and two minima of the symmetric structure $\left(\theta=0^{\circ}\right)$. Figures $3(\mathrm{a})$ and 3 (c) depict the contour profiles of fields $\left|H_{y}\right|$ for transmission maxima at $1235 \mathrm{~nm}$ and $665 \mathrm{~nm}$, respectively. According to (1) and (2), transmission maxima at wavelength $1235 \mathrm{~nm}$ and $665 \mathrm{~nm}$ correspond to the even order resonant mode of $N=2$ and 4, respectively. At the same time, joints between the ring cavity and two straight MIM waveguides are antinodes, and there is a higher magnetic field intensity in two straight MIM waveguides. It indicates that even-order waveguide resonances in the ring can lead to efficient coupling of the incident electromagnetic wave from MIM waveguide I through the ring cavity into MIM waveguide II. On the contrast, in Figures 3(b) and 3(d), the magnetic field distributions of transmission minima at $861 \mathrm{~nm}$ and $523 \mathrm{~nm}$ in the ring also produced a typical standing wave pattern, but the resonant mode numbers inside the ring are odd, such as $N=3$ and 5 . Because the MIM waveguide I and II symmetrically locate on both sides of the ring, the magnetic field distribution at joints of ring resonator with two straight MIM waveguide will be different: one is a node, and another is an antinode. Therefore, in such odd-order resonance condition, the incident electromagnetic wave from MIM waveguide I is strongly reflected back by the ring cavity, and the electromagnetic energy flow coupled into the ring cavity is relatively low. However, because the structure is symmetric, resulting in equivalent optical path in the ring, a certain optical transmission can be still maintained. Between the wavelength range of the maximum and minimum transmissions as the above mentioned, we can speculate that electromagnetic wave in the ring will not form a stable resonance (including even-order and odd-order resonance), and the optical transmission will vary between the maximum and minimum transmission.
Thirdly, we investigated the reason why there is a transmission minimum for most of the asymmetric structures $\left(\theta \neq 0^{\circ}\right)$ at the slightly bigger peak wavelength than one at which the transmission peak appears for the symmetric structure $\left(\theta=0^{\circ}\right)$. In addition, we also calculated the electromagnetic field distribution corresponding to the transmission minima. Figures 4(a)-4(c) depict the field distributions of $\left|H_{y}\right|$ for the asymmetric structure with $\theta=45^{\circ}$ at the transmission minimum wavelengths $1367 \mathrm{~nm}, 702 \mathrm{~nm}$, and $483 \mathrm{~nm}$, respectively. In Figure 4, one can find that the typical standing wave patterns formed in the ring and the antinode number inside the ring are even, which suggests that these wavelengths, respectively, correspond to the resonant modes for $N=2,4$, and 6 . However, the two straight MIM waveguides are asymmetric to the ring cavity, so the magnetic field distribution is different at the two joints. The joint with the waveguide I locates in the range of the magnetic antinode of standing wave in the ring resonator (not strictly on the location of the maximum of the magnetic intensity), so the magnetic field intensity at the joint is relatively strong. On the contrary, the magnetic field intensity at the joint with the waveguide II is weaker, just because the joint is the magnetic nodes of the standing wave. Therefore, only little electromagnetic wave of that joint can enter into the straight MIM waveguide II, resulting in a very low transmission. According to the relation between the positional deviation angle $\theta$ and the position of the magnetic nodes of the standing wave, it is not hard to forecast that the transmittance of the asymmetric structure with $\theta=45^{\circ}$ for the $N=4$ resonance mode could be close to zero, because the joint of the waveguide II and the ring may be locate at the magnetic nodes of the standing wave. Figures 2 and 4(b) can further confirm this point.

Fourthly, we also calculated the electromagnetic field distribution corresponding to the transmission maxima of the asymmetric structures $\left(\theta \neq 0^{\circ}\right)$. Figures $5(\mathrm{a})-5(\mathrm{c})$ show the contour profiles of magnetic field intensity $\left|H_{y}\right|$ at wavelengths $1206 \mathrm{~nm}(N=2$ mode $)$ for $\theta=45^{\circ}, 648 \mathrm{~nm}(N=$ 4 mode) for $\theta=30^{\circ}$, and $457 \mathrm{~nm}\left(N=6\right.$ mode) for $\theta=90^{\circ}$, respectively. In these pictures, one can see that magnetic field distribution of these transmission maxima has the same characteristic that the magnetic field intensities in the joints of the ring cavity with two straight MIM waveguides are relatively strong. All the joints are nearby antinodes of standing wave in the ring, which indicates that more electromagnetic wave from straight MIM waveguide I can couple into the ring cavity and enter into the straight MIM waveguide II.

Finally, we studied the influence of the radius of the ring on the wavelengths of the resonant mode. Figure 6 shows the transmission spectra corresponding to the rings with radii $r=135 \mathrm{~nm}, 155 \mathrm{~nm}$, and $175 \mathrm{~nm}$ for the symmetric structure $\left(\theta=0^{\circ}\right)$, respectively. As shown in Figure 6, the transmission peaks exhibit red shift as the radius increases. This result can be explained in terms of (1) and (2). According to (2), when $\varepsilon_{d}$ and $w$ are fixed, $n_{\text {eff }}$ changes little as $\lambda$ increases in the considered wavelength region. Consequently, $\lambda$ will increase as $r$ increases in terms of (1). In addition, we also calculated the transmission spectra of the MIM plasmonic waveguide with a ring resonator of different radius for different $\theta$. 


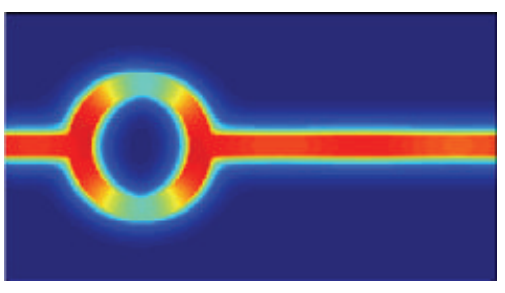

(a)

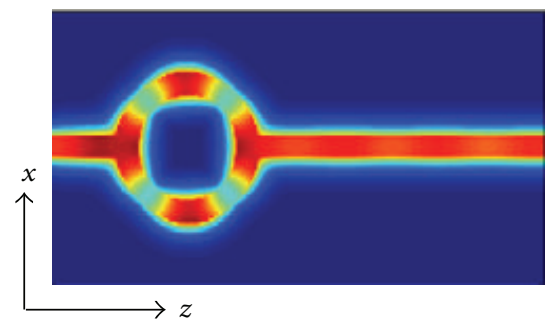

(c)

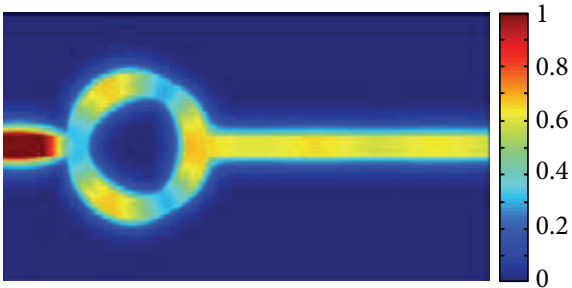

(b)

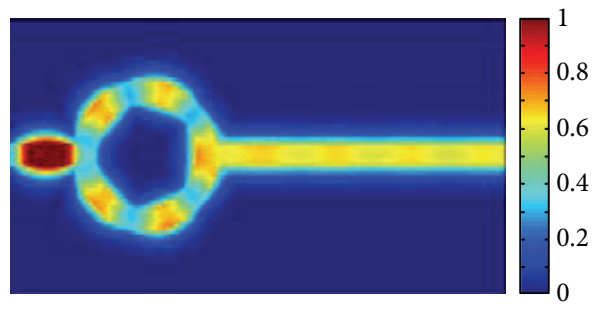

(d)

Figure 3: (Color Online) The contour profiles of magnetic field intensity $\left|H_{y}\right|$ at maximum and minimum wavelengths for the symmetric structure $\left(\theta=0^{\circ}\right)$. (a) and (c) are at maximum wavelengths $1235 \mathrm{~nm}$ and $665 \mathrm{~nm}$, respectively. (b) and (d) are at minimum wavelengths $861 \mathrm{~nm}$ and $523 \mathrm{~nm}$, respectively.

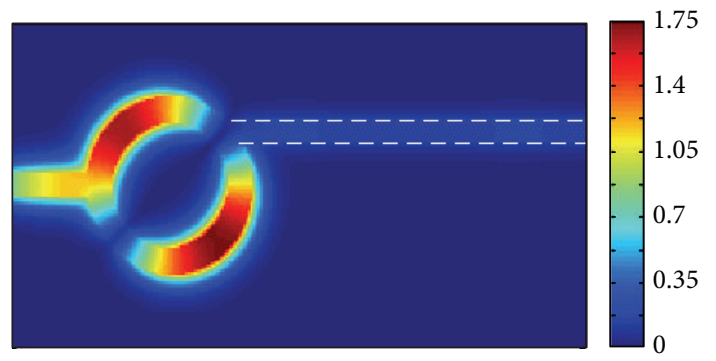

(a)

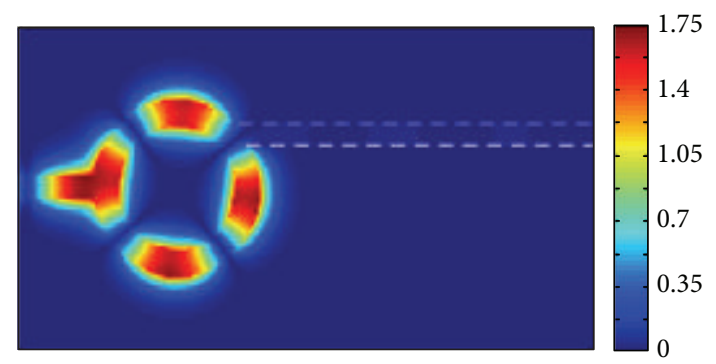

(b)

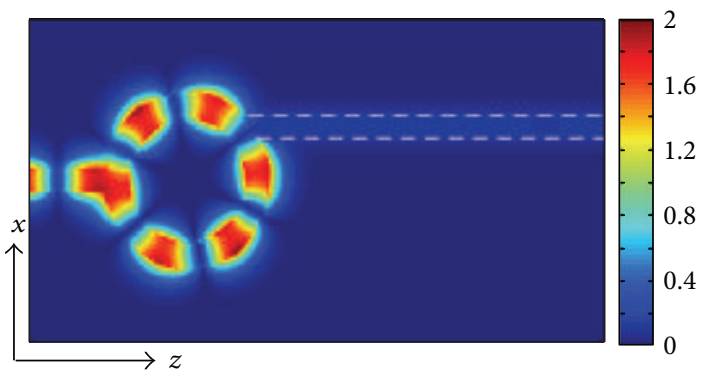

(c)

Figure 4: (Color Online) The contour profiles of magnetic field intensity $\left|H_{y}\right|$ at minimum wavelengths for the asymmetric structure with $\theta=45^{\circ}$. (a), (b), and (c) are at wavelengths $1367 \mathrm{~nm}, 702 \mathrm{~nm}$, and $483 \mathrm{~nm}$, respectively.

The result shows the transmission characteristic in these spectra is completely the same as that in Figure 2. Therefore, if we change the ring cavity radius, the wavelength of the maximum and minimum transmission discussed above will vary with the change of the radius of the ring resonator. This means that we can filter any wavelength by setting the geometric parameters of the structure.

\section{Summary}

In conclusion, the transmission characteristics of the electromagnetic wave are investigated in two-dimensional compound plasmonic structures composed of two straight subwavelength MIM waveguides and a ring resonator by using FDTD method. The results show that the filtering 


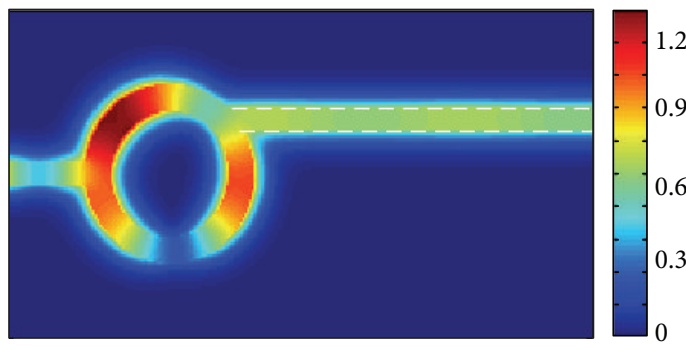

(a)

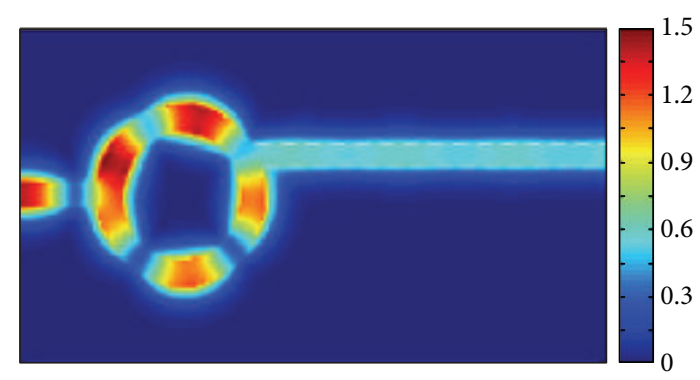

(b)

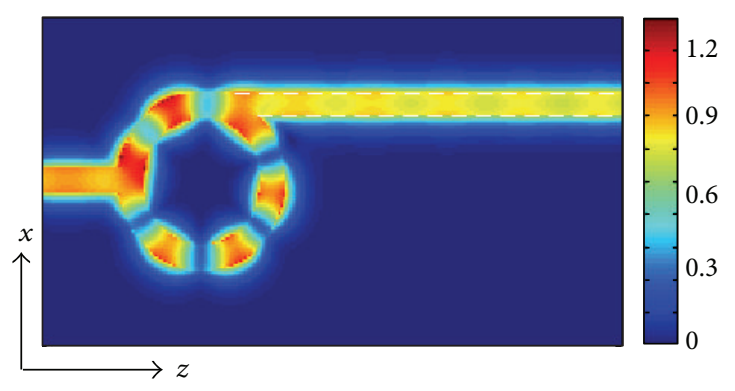

(c)

Figure 5: (Color Online) The contour profiles of magnetic field intensity $\left|H_{y}\right|$ at maximum wavelengths for the asymmetric structure. (a), (b), and (c) are at wavelengths $1206 \mathrm{~nm}$ for $\theta=45^{\circ}, 648 \mathrm{~nm}$ for $\theta=30^{\circ}$, and $457 \mathrm{~nm}$ for $\theta=90^{\circ}$, respectively.

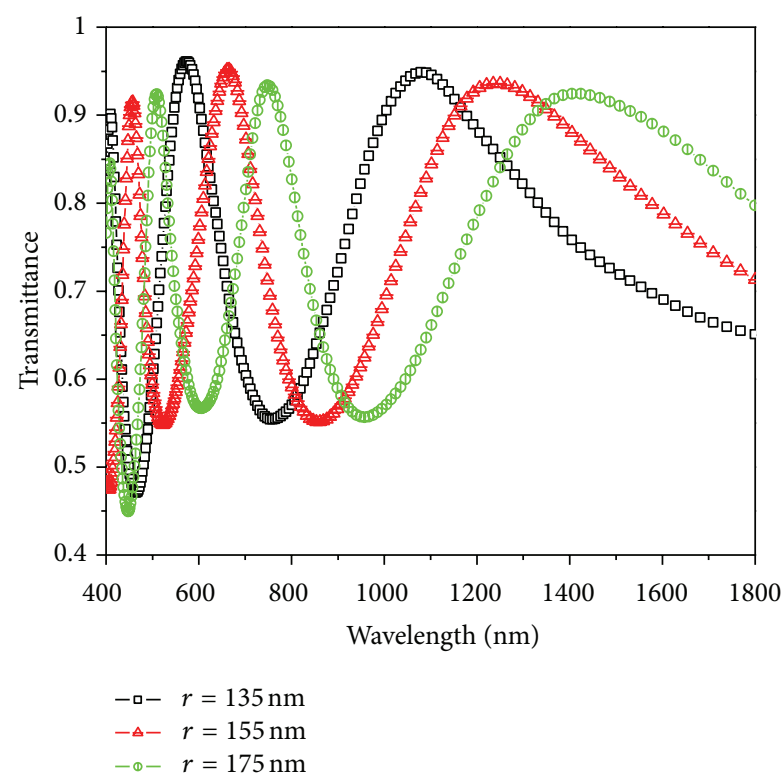

FIgUre 6: (Color Online) Transmission spectra of the MIM plasmonic waveguide with a different radius' ring resonator for $\theta=0^{\circ}$.

performances of the asymmetric structures (positional angular deviation $\theta \neq 0^{\circ}$ ) are greatly improved in comparison with the symmetric structure $\left(\theta=0^{\circ}\right)$. For the most of the asymmetric structures, there is a transmission minimum at the slightly bigger wavelength than that at which the transmission peak appears for the symmetric structures, and there is still a transmission peak at the wavelength of the transmission peak of the symmetric structures. And then the difference between the transmission peak and valley is increased, the breadth of the transmission peak becomes narrow. For these transmission minima of the asymmetric structures, it is found that the parts of coupling the MIM waveguide of the outgoing side with the ring resonator locate at the magnetic nodes of the standing waves in the ring resonator.

\section{Acknowledgments}

This work was supported by the National Natural Science Foundation of China (Grant nos. 11074069 and 61176116), the Specialized Research Fund for the Doctoral Program of Higher Education of China (Grant no. 20120161130003), the Hunan Provincial Natural Science Foundation of China (Grant no. 12JJ4009), the Hunan Provincial Science and Technology Project of China (Grant no. 2012FJ4121), and Aid program for Science and Technology Innovative Research Team in Higher Educational Institutions of Hunan Province.

\section{References}

[1] P. Berini, "Long-range surface plasmon polaritons," Advances in Optics and Photonics, vol. 1, no. 3, pp. 484-588, 2009.

[2] G. Veronis and S. Fan, "Bends and splitters in metal-dielectricmetal subwavelength plasmonic waveguides," Applied Physics Letters, vol. 87, no. 13, Article ID 131102, pp. 1-3, 2005.

[3] H. Gao, H. Shi, C. Wang et al., "Surface plasmon polariton propagation and combination in Y-shaped metallic channels," Optics Express, vol. 13, no. 26, pp. 10795-10800, 2005. 
[4] B. Wang and G. P. Wang, "Surface plasmon polariton propagation in nanoscale metal gap waveguides," Optics Letters, vol. 29, no. 17, pp. 1992-1994, 2004.

[5] Z. Han, L. Liu, and E. Forsberg, "Ultra-compact directional couplers and Mach-Zehnder interferometers employing surface plasmon polaritons," Optics Communications, vol. 259, no. 2, pp. 690-695, 2006.

[6] A. Hosseini and Y. Massoud, "A low-loss metal-insulator-metal plasmonic bragg reflector," Optics Express, vol. 14, no. 23, pp. 11318-11323, 2006.

[7] J. Q. Liu, L. L. Wang, M. D. He et al., "A wide bandgap plasmonic Bragg reflector," Optics Express, vol. 16, no. 7, pp. 4888-4894, 2008.

[8] T. Nikolajsen, K. Leosson, and S. I. Bozhevolnyi, "Surface plasmon polariton based modulators and switches operating at telecom wavelengths," Applied Physics Letters, vol. 85, no. 24, pp. 5833-5835, 2004.

[9] H. Zhao, X. G. Guang, and J. Huang, "Novel optical directional coupler based on surface plasmon polaritons," Physica E, vol. 40, no. 10, pp. 3025-3029, 2008.

[10] X. S. Lin and X. G. Huang, "Tooth-shaped plasmonic waveguide filters with nanometeric sizes," Optics Letters, vol. 33, no. 23, pp. 2874-2876, 2008.

[11] T. B. Wang, X. W. Wen, C. P. Yin, and H. Z. Wang, "The transmission characteristics of surface plasmon polaritons in ring resonator," Optics Express, vol. 17, no. 26, pp. 24096-24101, 2009.

[12] D. Xiang, L. L. Wang, L. Wang et al., "Optical transmission through double-layer compound metallic gratings with subwavelength slits," Journal of Modern Optics, vol. 59, no. 15, pp. 1342-1348, 2012.

[13] A. Taflove and S. C. Hagness, Computational Electrodynamics: The Finite-Difference Time-Domain Method, Artech House, Norwood, Mass, USA, 2nd edition, 2000.

[14] D. Xiang, L. L. Wang, X. F. Li et al., "Transmission resonances of compound metallic gratings with two subwavelength slits in each period," Optics Express, vol. 19, no. 3, pp. 2187-2192, 2011. 

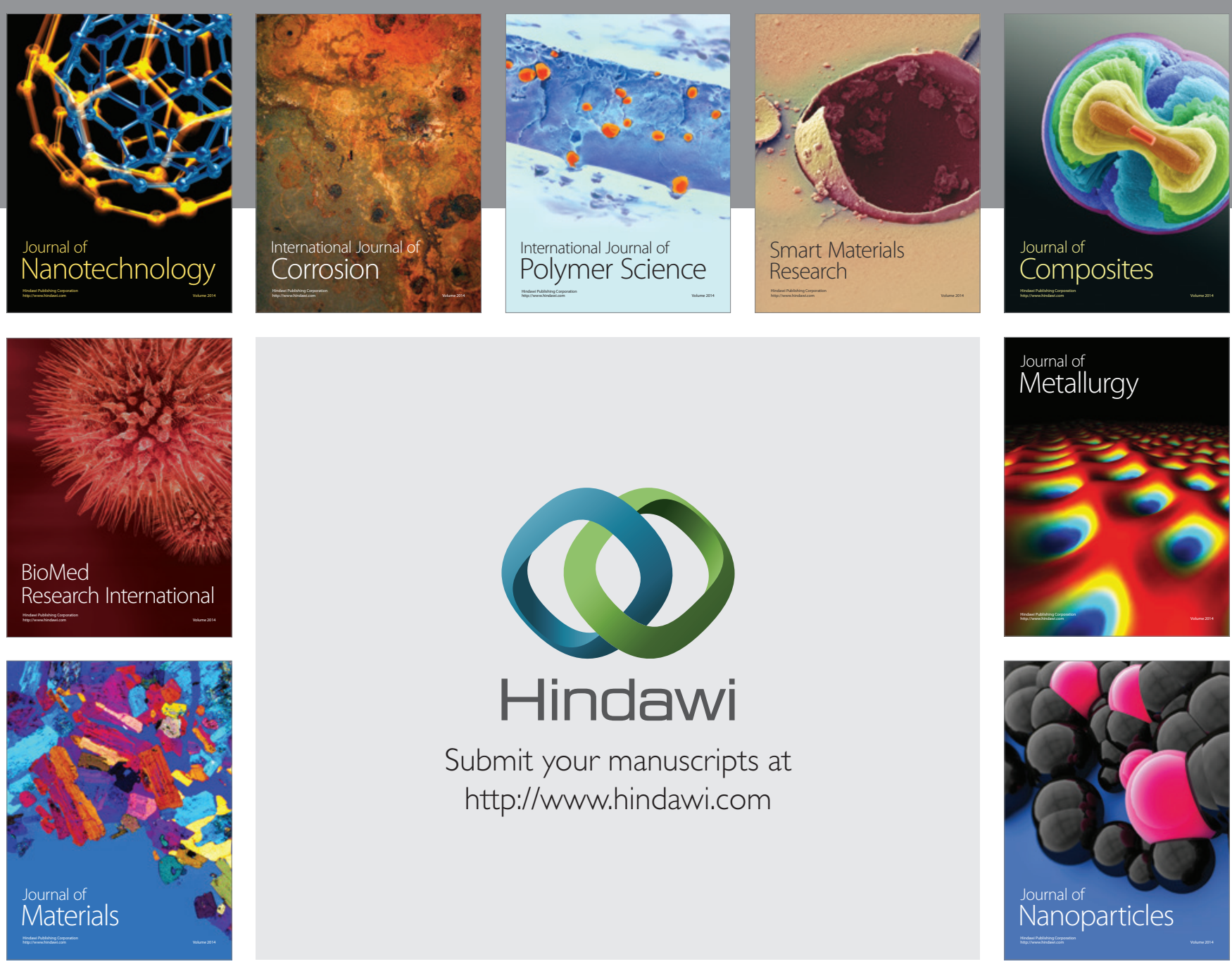

Submit your manuscripts at http://www.hindawi.com
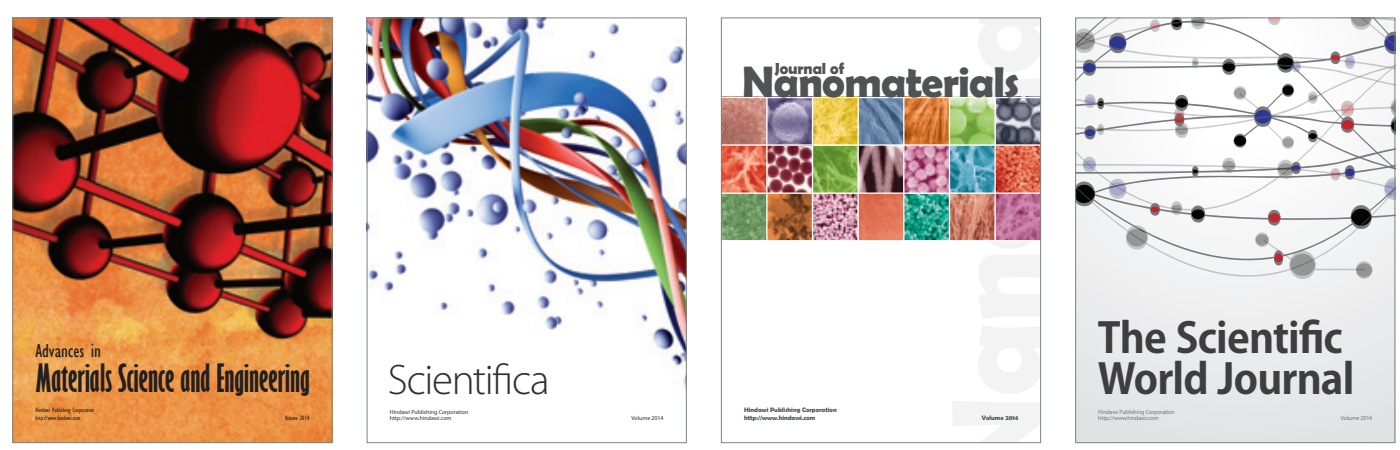

\section{The Scientific World Journal}
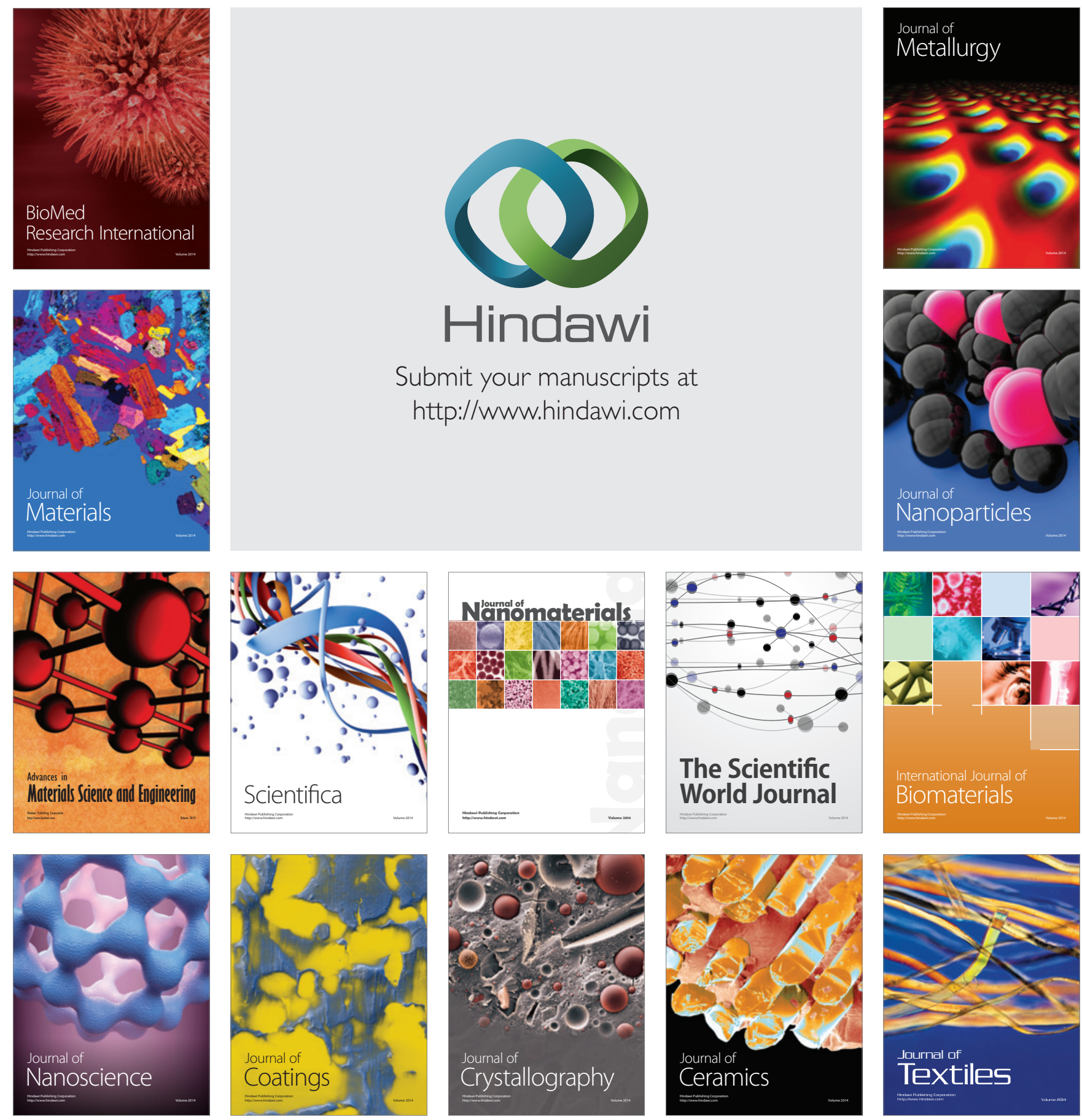